\title{
Impact of COVID-19 on the pain and disability of patients with adult spinal deformity
}

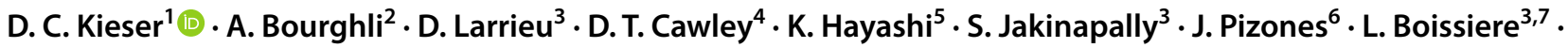 \\ I. Obeid ${ }^{3,7}$
}

Received: 16 October 2020 / Accepted: 16 February 2021 / Published online: 2 March 2021

(c) Scoliosis Research Society 2021

\begin{abstract}
Purpose To evaluate the pain and functional effect of the COVID-19 pandemic on patients with ASD reflected by their response to SRS-22, ODI, and SF-36 questionnaires.

Methods Patients who had stable pain and functional outcome scores over the preceding 2 years were enrolled in a local prospectively collected adult spinal deformity (ASD) database. A reanalysis of their SRS22, ODI and SF-36 data 14 days into confinement were compared to their last pre-confinement scores.

Results 89 patients were included in this study (average age 60.7 years, $91 \%$ female) with an average time from last FU until confinement of 9.6 months. The ODI total score worsened by 5 points post-confinement with no difference seen in personal care, walking and social life. In contrast, the SRS-22 score showed small improvements in function/activity and satisfaction, but no significant differences for the other domains. Similarly, the SF-36 showed small improvements in physical function, physical and emotional role, vitality and PCS.

Conclusion The global COVID-19 pandemic and ensuing confinement had variable overall effects on ASD patients, without the expected marked worsening. In addition, this study illustrates that the SRS-22 questionnaire is less influenced by environmental and psychological factors than the ODI supporting its objectivity and accuracy in the evaluation of the QoL of ASD patients.
\end{abstract}

Keywords Spine $\cdot$ COVID- $19 \cdot$ Coronavirus $\cdot$ Surgery $\cdot$ Scoliosis

D. C. Kieser

kieserdavid@gmail.com

1 Department of Orthopaedic Surgery and Musculoskeletal Medicine, University of Otago, Christchurch School of Medicine, 2 Riccarton Avenue, Christchurch 8011, New Zealand

2 Orthopedic and Spinal Surgery Department, Kingdom Hospital, P.O. Box 84400, Riyadh 11671, Saudi Arabia

3 L'Institut de la Colonne Vertébrale, CHU Pellegrin, 33076 Bordeaux, France

4 Mater Private Hospital, Dublin, Ireland

5 Department of Orthopedic Surgery, Osaka City University Graduate School of Medicine, Osaka, Japan

6 Spine Unit, Hospital La Paz, Madrid, Spain

7 ELSAN, Polyclinique Jean Villar, Avenue Maryse Bastie, 33520 Bruges, France

\section{Introduction}

Spinal conditions are a major cause of pain and disability worldwide. Chronic spinal pathology, such as adult spinal deformity (ASD) is common, affecting a third of patients aged over 50 years and two-thirds of patients aged over 70 years [1]. ASD is known to affect the quality of life (QoL), functional capacity and pain of patients [2, 3]. In fact, ASD has the worst patient reported QoL of all common chronic medical conditions, including arthritis, chronic lung disease, congestive heart failure, diabetes and ischaemic heart disease [4]. However, various factors may influence the degree of this disability and QoL including psychological distress [5-7].

2020 saw a pandemic infection from COVID-19. The effect of this pandemic is far reaching and includes marked psychological distress to populations worldwide [8-10]. In fact, COVID 19 has incited such fear that new scales, such as the fear of COVID-19 scale, have been necessitated 
to understand a population's phobia [11]. This includes patients personally unaffected by the virus.

Clearly, the psychological effect of COVID-19 has also affected patients with ASD, even in those not personally infected. This pandemic has, therefore, provided an unprecedented opportunity to further understand the effect of a global event on the pain, function and QoL of patients with ASD. It may be predicted that the overwhelming psychological effect of COVID-19 may improve pain and function in these patients as their perspective shifts from their spinal pathology to a more imminent risk. Alternatively, the stress exerted by COVID-19 may exacerbate symptoms. Compounding this, populations were confined to their homes, permitting less physical exertion on their spinal deformity, but potentially more sedentary time to focus on their health.

Thus, the aim of this study was to determine the effect of the COVID-19 pandemic on the pain, function and QoL of patients with ASD not personally infected by COVID19 in France during confinement, and in doing so, to explore whether variations in responses to questionnaires in the undercurrent condition are affected by non-spinerelated issues.

\section{Methods}

Consecutive patients included in this study were extracted from a prospective database of patients who had previously undergone deformity correction for ASD. The inclusion criteria were: patients with degenerative or idiopathic ASD, defined as a coronal Cobb angle $>20^{\circ}$, thoracic kyphosis $>60^{\circ}$, sagittal vertical axis (SVA) $>5 \mathrm{~cm}$ or pelvic tilt $(\mathrm{PT})>25^{\circ}$ treated with deformity correction; a minimum of 2 years follow-up post-surgery, with "clinical stability" during the last two follow-ups prior to confinement and the last follow-up visit being within 1 year of the start of confinement. Patients were excluded if they had been previously or were currently diagnosed as COVID19 positive.

"Clinical stability" over the last two follow-ups was defined using the Oswestry Disability Index (ODI), Scoliosis Research Society 22 (SRS-22) and Short Form 36 (SF36) Physical Component Score (PCS). Only patients with a difference in ODI less than or equal to 15 points [ODI last follow-up (FU)-ODI previous FU] and a variation in the absolute value of SRS-22 total score and SF-36 PCS [(Last FU-Previous FU)/Previous FU] less than or equal to $20 \%$ were considered to have "clinical stability".

All patients were from the same geographical area, affected by the same governmental restrictions and answered all three questionnaires within 15 days of confinement.

\section{Statistical analysis}

Cross-tabulation was generated and either a Fisher exact or Pearson $\mathrm{Chi}^{2}$ test was used to compare all the distributions. Paired $t$ tests were used to assess differences in means for the same cohort between different follow-up time points. A $p$ value $<0.05$ was considered statistically significant.

\section{Results}

89 patients were included in this analysis (average age 60.7 years (SD 16.8), $91 \%$ female). The average time from last FU until confinement was 9.6 months (SD 4.9). The comparison between the last pre-confinement FU and postconfinement FU is shown in Table 1.

Overall the ODI, which is an index derived to quantify disability from low back pain, was seen to worsen by five points post-confinement with no difference seen in personal care, walking and social life. In contrast, the SRS-22 score, which is the most widely used patient reported outcome score for spinal deformity, showed small improvements in function/activity and satisfaction, but no significant differences for the other domains. Similarly, the SF-36, which is a health-related quality of life score, showed small improvements in physical function, physical and emotional role, vitality and PCS.

\section{Discussion}

To our knowledge, this is the first study to assess the effects of a global pandemic on patients with ASD by analysing its consequences on health-related QoL scores. We hypothesised that confinement would have detrimental psychological impacts and a lack of rehabilitation that would result in patients experiencing worsened pain, function and QoL. This hypothesis was confirmed when using the ODI, with the overall score, pain, lifting, sitting, standing, sleeping, sex life and travelling all being rated statistically $(p<0.05)$ and clinically ( $>10 \%$ change) significantly poorer during confinement. Furthermore, an unchanged social life on ODI scores does not correlate with the societal effects of confinement during this time.

However, both the SRS-22 and SF-36 questionnaire had no significant change in the patient's reported pain and showed improvements in the SRS-22 function and satisfaction, as well as the SF-36 physical function, emotion and vitality. This contrasted our expectation and necessitates a reconsideration of the detrimental effect of confinement during a global crisis. In addition, it may raise the question 
Table 1 Comparison of outcome scores pre- and post-confinement

\begin{tabular}{|c|c|c|c|c|}
\hline & Mean & $N$ & SD & $P$ \\
\hline \multicolumn{5}{|l|}{ ODI } \\
\hline Pain intensity & & & & 0.003 \\
\hline Last follow-up & 1.1 & 89 & 1.1 & \\
\hline Post confinement & 1.4 & 89 & 1.3 & \\
\hline Personal care & & & & 0.661 \\
\hline Last follow-up & 0.4 & 89 & 0.9 & \\
\hline Post confinement & 0.5 & 89 & 1.0 & \\
\hline Lifting & & & & 0.000 \\
\hline Last follow-up & 2.3 & 89 & 1.5 & \\
\hline Post confinement & 3.2 & 89 & 1.7 & \\
\hline Walking & & & & 0.680 \\
\hline Last follow-up & 0.9 & 89 & 1.3 & \\
\hline Post confinement & 0.9 & 89 & 1.3 & \\
\hline Sitting & & & & 0.014 \\
\hline Last follow-up & 0.8 & 88 & 1.0 & \\
\hline Post confinement & 1.1 & 88 & 1.4 & \\
\hline Standing & & & & 0.002 \\
\hline Last follow-up & 1.3 & 89 & 1.3 & \\
\hline Post confinement & 1.9 & 89 & 1.7 & \\
\hline Sleeping & & & & 0.043 \\
\hline Last follow-up & 0.5 & 89 & 0.8 & \\
\hline Post confinement & 0.8 & 89 & 1.3 & \\
\hline Sex life & & & & 0.004 \\
\hline Last follow-up & 0.3 & 45 & 0.7 & \\
\hline Post confinement & 0.8 & 45 & 1.6 & \\
\hline Social life & & & & 0.171 \\
\hline Last follow-up & 1.0 & 89 & 1.3 & \\
\hline Post confinement & 0.8 & 89 & 1.2 & \\
\hline Travelling & & & & 0.025 \\
\hline Last follow-up & 0.9 & 89 & 1.1 & \\
\hline Post confinement & 1.2 & 89 & 1.6 & \\
\hline Total score & & & & 0.000 \\
\hline Last follow-up & 20.1 & 89 & 15.2 & \\
\hline Post confinement & 25.8 & 89 & 20.5 & \\
\hline \multicolumn{5}{|l|}{ SRS-22 } \\
\hline Function/activity & & & & 0.016 \\
\hline Last follow-up & 3.9 & 89 & 0.9 & \\
\hline Post confinement & 4.1 & 89 & 0.7 & \\
\hline Pain & & & & 0.879 \\
\hline Last follow-up & 3.7 & 89 & 1.0 & \\
\hline Post confinement & 3.8 & 89 & 1.0 & \\
\hline Self-image/appearance & & & & 0.052 \\
\hline Last follow-up & 3.8 & 89 & 0.8 & \\
\hline Post confinement & 3.7 & 89 & 0.9 & \\
\hline Mental health & & & & 0.220 \\
\hline Last follow-up & 3.8 & 89 & 0.9 & \\
\hline Post confinement & 3.9 & 89 & 0.9 & \\
\hline Satisfaction with management & & & & 0.004 \\
\hline Last follow-up & 4.4 & 89 & 0.7 & \\
\hline Post confinement & 4.6 & 89 & 0.7 & \\
\hline
\end{tabular}

Table 1 (continued)

\begin{tabular}{|c|c|c|c|c|}
\hline & Mean & $N$ & SD & $P$ \\
\hline Total score & & & & 0.314 \\
\hline Last follow-up & 3.9 & 89 & 0.7 & \\
\hline Post confinement & 3.9 & 89 & 0.7 & \\
\hline \multicolumn{5}{|l|}{ SF-36 } \\
\hline Physical function & & & & 0.012 \\
\hline Last follow-up & 44.9 & 89 & 9.2 & \\
\hline Post confinement & 46.5 & 89 & 8.8 & \\
\hline Physical role & & & & 0.003 \\
\hline Last follow-up & 45.8 & 89 & 10.9 & \\
\hline Post confinement & 49.7 & 89 & 10.9 & \\
\hline Pain & & & & 0.058 \\
\hline Last follow-up & 46.5 & 89 & 9.3 & \\
\hline Post confinement & 48.2 & 89 & 11.0 & \\
\hline Vitality & & & & 0.001 \\
\hline Last follow-up & 49.4 & 89 & 10.1 & \\
\hline Post confinement & 46.0 & 89 & 9.9 & \\
\hline Social functioning & & & & 0.768 \\
\hline Last follow-up & 48.9 & 89 & 9.8 & \\
\hline Post confinement & 48.6 & 89 & 11.4 & \\
\hline Emotional role & & & & 0.000 \\
\hline Last follow-up & 47.1 & 89 & 11.5 & \\
\hline Post confinement & 52.5 & 89 & 8.6 & \\
\hline Mental health & & & & 0.569 \\
\hline Last follow-up & 48.5 & 89 & 10.5 & \\
\hline Post confinement & 49.1 & 89 & 11.9 & \\
\hline General health & & & & 0.051 \\
\hline Last follow-up & 51.9 & 89 & 9.2 & \\
\hline Post confinement & 53.9 & 89 & 1.0 & \\
\hline PCS & & & & 0.024 \\
\hline Last follow-up & 46.6 & 89 & 9.2 & \\
\hline Post confinement & 48.4 & 89 & 10.1 & \\
\hline MCS & & & & 0.555 \\
\hline Last follow-up & 49.6 & 89 & 10.5 & \\
\hline Post confinement & 50.3 & 89 & 9.6 & \\
\hline
\end{tabular}

$P<0.05$ (in bold)

of the specificity of each score as an objective indicator of the QoL of spinal deformity patients [12-14]. Considering the "clinical stability" of these patients, the psychological influence of the pandemic and staying at home during the lockdown period can be considered the main factors that influenced any modification in the score results.

Clearly, these results cannot be extrapolated to times when normal daily activity is feasible and does not represent potential benefits for patients to remain at home permanently, but rather offers insight into the effect of the COVID-19 pandemic on the ASD community. In addition, this study cannot be extrapolated to patients affected by back pain without underlying deformity, where the interpretation 
would wrongly suggest potential benefits of remaining at home.

In addition, this study has a multitude of limitations that need to be recognised when interpreting the results. First, only operative patients were included. Second, despite the previous 2 years FU showing stable scores and it being felt unlikely that the spinal related complications arose during the time between latest FU and confinement, particularly considering that complications would have impacted all three questionnaires simultaneously, radiological follow-up was not performed to assess for complications [14]. Third, there is no stratification of age, pulmonary status or radiographic severity of spinal deformity that could be influenced by the COVID-19 infections. Fourth, this is a single-centre study, therefore, differences among other countries and territories due to the differences of the COVID-19 pandemic need to be considered. Lastly, we did not correlate the outcome scores to any psychological well-being score.

\section{Conclusion}

The global COVID-19 pandemic and ensuing confinement had variable overall effects on patients, without the expected marked worsening. In addition, this study illustrates a higher specificity for the SRS-22 questionnaire when compared to the ODI in assessing ASD patients with less influence from environmental or psychological factors, emphasising its objectivity and accuracy in the evaluation of the QoL of ASD patients.

\section{Acknowledgements Glynny Kieser for her editorial input.}

Author contributions DCK: acquisition, conception, design, analysis, interpretation, draft, critical revision, approved version, agree to be accountable for all aspects of the work in ensuring that questions related to the accuracy or integrity of any part of the work are appropriately investigated and resolved; $\mathrm{AB}, \mathrm{IO}$ : acquisition of data, design, critical revision, approved version, agree to be accountable for all aspects of the work in ensuring that questions related to the accuracy or integrity of any part of the work are appropriately investigated and resolved; DTC, DL, KH, SJ, JP, LB: acquisition of data, critical revision, approved version, agree to be accountable for all aspects of the work in ensuring that questions related to the accuracy or integrity of any part of the work are appropriately investigated and resolved.

Funding No funding was received for this work.

Data availability On request from the corresponding author.

\section{Declaration}

Ethical approval This study was approved by the IRB of Pellegrin Hospital (CE-GP-2020-31) and the study was performed in accordance with the ethical standards as laid down in the 1964 Declaration of Helsinki.
Consent to participate Informed consent was obtained from all individual participants included in the study.

\section{References}

1. Schwab F, Dubey A, Gamez L, El Fegoun AB, Hwang K, Pagala $\mathrm{M}$ et al (2005) Adult scoliosis: prevalence, SF-36, and nutritional parameters in an elderly volunteer population. Spine 30(9): 1082-1085

2. Schwab F, Dubey A, Pagala M, Gamez L, Farcy JP (2003) Adult scoliosis: a health assessment analysis by SF-36. Spine 28(6):602-606

3. Schwab FJ, Blondel B, Bess S, Hostin R, Shaffrey CI, Smith JS et al (2013) Radiographical spinopelvic parameters and disability in the setting of adult spinal deformity: a prospective multicenter analysis. Spine 38(13):E803-E812

4. Pellise F, Vila-Casademunt A, Ferrer M, Domingo-Sabat M, Bago J, Perez-Grueso FJ et al (2015) Impact on health related quality of life of adult spinal deformity (ASD) compared with other chronic conditions. Eur Spine J 24(1):3-11

5. Hurwitz EL, Morgenstern H, Yu F (2003) Cross-sectional and longitudinal associations of low-back pain and related disability with psychological distress among patients enrolled in the UCLA low back pain study. J Clin Epidemiol 56(5):463-471

6. Theologis AA, Ailon T, Scheer JK, Smith JS, Shaffrey CI, Bess S et al (2016) Impact of preoperative depression on 2-year clinical outcomes following adult spinal deformity surgery: the importance of risk stratification based on type of psychological distress. J Neurosurg Spine 25(4):477-485

7. Bakhsheshian J, Scheer JK, Gum JL, Hostin R, Lafage V, Bess S et al (2017) Impact of poor mental health in adult spinal deformity patients with poor physical function: a retrospective analysis with a 2-year follow-up. J Neurosurg Spine 26(1):116-124

8. Cao W, Fang Z, Hou G, Han M, Xu X, Dong J, Zheng J (2020) The psychological impact of the COVID-19 epidemic on college students in China. Psych Res 287:112934

9. Wang C, Pan R, Wan X, Tan Y, Xu L, Ho CS, Ho RC (2020) Immediate psychological responses and associated factors during the initial stage of the 2019 Coronavirus Disease (COVID-19) epidemic among the general population in China. Int J Environ Res 17:1729

10. Cullen W, Gulati G, Kelly BD (2020) Mental health in the COVID19 pandemic. Int J Med 113(5):311-312

11. Ahorsu DK, Lin CY, Imani V, Saffari M, Griffiths MD, Pakpour AH (2020) The fear of COVID-19 scale: development and initial validation. Int J Mental Health Addict. https://doi.org/10.1007/s1146 9-020-00270-8

12. Fujishiro T, Boissière L, Cawley DT, Larrieu D, Gille O, Vital JM (2019) Adult spinal deformity surgical decision-making score: Part 1: development and validation of a scoring system to guide the selection of treatment modalities for patients below 40 years with adult spinal deformity. Eur Spine J 28(7):1652-1660

13. Fujishiro T, Boissière L, Cawley DT, Larrieu D, Gille O, Vital JM (2020) Adult spinal deformity surgical decision-making score. Part 2: development and validation of a scoring system to guide the selection of treatment modalities for patients above 40 years with adult spinal deformity. Eur Spine J 29(1):45-53

14. Boissière L, Takemoto M, Bourghli A, Vital JM, Pellisé F, Alanay A (2017) Global tilt and lumbar lordosis index: two parameters correlating with health-related quality of life scores-but how do they truly impact disability? Spine J 17(4):480-488

Publisher's Note Springer Nature remains neutral with regard to jurisdictional claims in published maps and institutional affiliations. 